\title{
Small Molecule Microcrystal Electron Diffraction (MicroED) for the Pharmaceutical Industry
}

\author{
J Bruhn ${ }^{1}$, A Cheng ${ }^{1}$, T Nieusma ${ }^{1}$, S Dallakyan ${ }^{1}$, J Speir $^{1}$, A Schneemann ${ }^{1}$, C Potter ${ }^{1}$, B Carragher ${ }^{1}$, \\ G Scapin ${ }^{1}$ \\ ${ }^{1}$ Nanolmaging Services, San Diego, CA, USA \\ jbruhn@nanoimagingservices.com
}

The emerging field of microcrystal electron diffraction (microED) is of great interest to the pharmaceutical industry. The promise of high-resolution structures without the need to grow large, single crystals makes this technique very attractive as it lowers sample requirements and in many cases eliminates the need for crystallization trials. Here we will present our experience collecting microED data from over 30 small molecule samples for clients across the pharmaceutical industry. We have adapted the TEM data acquisition software package Leginon to collect high-quality diffraction data in an automated manner. We have used this data acquisition pipeline coupled to our DIALS-based data reduction pipeline to solve over 20 small molecule crystal structures in-house. We will present some of the unique challenges posed by small molecule microED data processing and we will share our experience adapting tools built for processing protein diffraction data to particularly challenging cases. We hope to inspire conversation amongst developers of data processing packages to improve the tools available to those venturing into the world of electron diffraction.

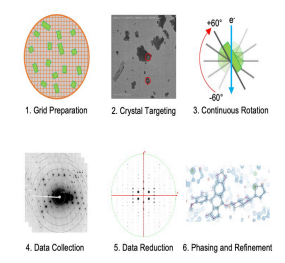

Figure 1

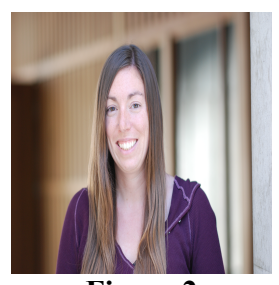

Figure 2

Acta Cryst. (2020). A76, a110 